\title{
Short communication: Enhanced autophagy activity in liver tissue of dairy cows with mild fatty liver
}

\author{
Meng Chen, ${ }^{1}$ Juan J. Loor, ${ }^{2} \odot$ Qian Zhai, ${ }^{3}$ Yusheng Liang, ${ }^{2} \odot$ Hao Yu, ${ }^{1}$ Xiliang Du, ${ }^{1}$ Taiyu Shen, ${ }^{1} \odot$ \\ Zhiyuan Fang, ${ }^{1}$ Zhen Shi, ${ }^{1}$ Xinghui Wang, ${ }^{1}$ Yiwei Zhu, ${ }^{1}$ Yuxiang Song, ${ }^{1}$ Guowen Liu,,${ }^{1} \odot$ Xiaobing Li, ${ }^{1}{ }^{\circ}$ \\ Zhe Wang, ${ }^{1}$ and Xinwei Li ${ }^{1 *}$ (i) \\ ${ }^{1}$ Key Laboratory of Zoonoses Research, Ministry of Education, College of Veterinary Medicine, Jilin University, 5333 Xi'an Road, Changchun, \\ Jilin Province, 130062, China \\ ${ }^{2}$ Mammalian NutriPhysioGenomics, Department of Animal Sciences and Division of Nutritional Sciences, University of Illinois, Urbana 61801 \\ ${ }^{3}$ College of Wildlife Resources, Northeast Forestry University, No. 26 Hexing Road, Xiangfang District, Harbin, Heilongjiang Province, 150040, \\ China
}

\section{ABSTRACT}

During the transition period, dairy cows are challenged by increased energy demands and decreased dry matter intake, which can induce a variety of metabolic disorders, especially fatty liver. Dairy cows suffering from mild or moderate fatty liver in this period show no distinct clinical symptoms, indicating the occurrence of adaptive processes. The process of autophagy (an adaptive response) leads to degradation of intracellular components to generate energy and maintains cellular homeostasis during negative nutrient status. Whether autophagy is involved in metabolic adaptations of the pathological course of mild fatty liver is unclear. Thus, the aim of this study was to determine hepatic autophagy status in dairy cows with mild fatty liver. Liver samples were collected from healthy cows ( $\mathrm{n}=$ 15), defined as having hepatic triglyceride (TG) content $<1 \%$ on a wet weight basis, and cows with mild fatty liver $(\mathrm{n}=15)$, defined as having hepatic TG content between 1 and $5 \%$. The abundance of the ubiquitinated proteins, microtubule-associated protein 1 light chain 3 (MAP1LC3, also called LC3-II) and sequestosome-1 (SQSTM1, also called p62) was lower, whereas the mRNA abundance of MAP1LC3 and SQSTM1 was greater in cows with mild fatty liver. The hepatic mRNA abundance of autophagy-related (ATG) genes $A T G 5$ and $A T G 7$ was greater in response to fatty liver. However, the protein abundance of ATG5 and ATG7 did not differ between healthy and mild fatty liver cows. Together, these data indicate that the formation and degradation of autophagosomes is enhanced in the liver of cows with mild fatty liver. Besides, these results

Received August 14, 2019.

Accepted December 3, 2019.

*Corresponding author: lixinwei100@126.com are conducive to define the adaptation mechanisms of dairy cows during the transition period.

Key words: mild fatty liver, metabolic adaptation, autophagy, MAP1LC3 (LC3-II)

\section{Short Communication}

The transition period in dairy cows, defined as 3 wk before to 3 wk after parturition, is characterized by dramatic physiological changes that impose severe challenges from the standpoint of energy balance (Ingvartsen, 2006; Loor et al., 2013). A key problem during transition is the tremendous increase in energy requirements for milk production that, paralleled with a decrease in feed intake, drives cows into a state of negative energy balance (NEB). As a result, the liver undergoes important metabolic adaptations designed to accommodate not only the influx of fatty acids but also the need for gluconeogenesis to support milk synthesis (Ha et al., 2017; Qin et al., 2018). Although several adaptive biochemical, transcriptional, and translational mechanisms experienced by the liver during the transition period have been reported (Loor et al., 2013; Imhasly et al., 2015; Du et al., 2018b), much less is known about the underlying mechanisms that allow hepatocytes to cope with the decrease in nutrition supply.

Fatty acids are energy-rich molecules (JuárezHernández et al., 2016) that can be catabolized in the liver to produce ATP (Lavallard and Gual, 2014). However, excessive and sustained body fat mobilization results in a sharp increase in the influx of fatty acids to the liver, often overwhelming its capacity for complete oxidation and export into lipoproteins (Litherland et al., 2011; Loor et al., 2013). Clearly, the predisposition of bovine liver to accumulate lipid with a sustained surge in the influx of fatty acids accounts for the high incidence of mild (triglyceride content in the liver of $1-5 \%$ ) or moderate (triglyceride content in the liver 
of $5-10 \%$ ) fatty liver in the first month after calving; incidences ranging from 30 to $40 \%$ have been reported (Bobe et al., 2004). Ceciliani et al. (2018) reported that cows with mild or moderate fatty liver had no distinct clinical symptoms and were able to navigate the transition period uneventfully; they concluded that metabolic adjustments beyond lipid metabolism play an important role during NEB.

Autophagy is a highly conserved recycling process involving the degradation of harmful or useless proteins and organelles in lysosomes (Ravanan et al., 2017). During negative nutrient status, autophagy provides substrates to sustain cellular metabolism and, hence, preserve tissue function (Kim and Lee, 2014). Dairy cows with mild fatty liver display energy deficiency (Bobe et al., 2004; Du et al., 2018b). Moreover, hepatic metabolic activity is enhanced in the dairy cows with mild fatty liver (Du et al., 2018b), which would produce more damaged or useless organelles and proteins. Thus, autophagy might be an effective way to offset the adverse effects of a prolonged NEB state and degrade damaged or useless organelles and proteins. Based on this, we hypothesize that autophagy might be enhanced in the liver of dairy cows experiencing mild fatty liver. Therefore, the objective of this study was to investigate hepatic autophagy status in cows with mild fatty liver.

All animal protocols in the present study were approved by the Ethics Committee on the Use and Care of Animals at Jilin University [Changchun, China; 2018 clinical trial (2018-12047)]. Animals received humane care according to the principles and specific guidelines presented in Guide for the Care and Use of Agricultural Animals in Research and Teaching (FASS, 2010). A physical examination of the cows in this study ensured the absence of co-morbidities. Cows were fed ad libitum once per day at $1100 \mathrm{~h}$, and the composition of the basal diet is shown in Supplemental Table S1 (https: //doi.org/10.3168/jds.2019-17457). Lactating Holstein cows with similar numbers of lactations (median $=3$, range $=2$ to 4 ) and DIM (median $=6 \mathrm{~d}$, range $=3$ to $9 \mathrm{~d}$ ) were selected from a 1,000-cow dairy farm located in Changchun, Jilin Province, China. Liver triglyceride (TG) content is the gold standard for diagnosing fatty liver in dairy cows (Bobe et al., 2004), and it was used to classify cows as healthy ( $\mathrm{TG}<1 \%, \mathrm{~g} / \mathrm{g}$ of wet weight) or as having mild fatty liver (TG 1-5\%). Fifteen cows with mild fatty liver and 15 healthy cows were selected via this approach.

Milk yield was recorded on 3 consecutive days at 0530 and $1500 \mathrm{~h}$. Blood samples were collected with 5 -mL vacuum collection tubes (no anticoagulant, Sanli Medical Technology Development Co. Ltd., Liuyang, China) on 3 consecutive days via jugular venipuncture between 0700 and $0800 \mathrm{~h}$, before feeding, and immedi- ately centrifuged at $3,500 \times g$ for 15 min at $4^{\circ} \mathrm{C}$. Serum was obtained and stored at $-80^{\circ} \mathrm{C}$ until analysis. Liver biopsies were harvested by an experienced veterinarian. First, the intercostal space was shaved. Then, iodine scrub and $75 \%$ alcohol were used to sanitize the incision area, followed by anesthesia with a subcutaneous injection of $2 \%$ lidocaine $\mathrm{HCl}$. A scalpel blade was used to make a 3-mm skin incision at the 11th intercostal space to allow insertion of a stainless steel trocar $(31 \mathrm{~cm}$ long and $7.5 \mathrm{~mm}$ in diameter) into the abdominal cavity, directing the point of the trocar toward the left elbow. Liver tissue (approximately $150 \mathrm{mg}$ ) was immediately frozen in liquid nitrogen. Cows were housed in a tiestall barn during the study. The description of the cows and the baseline characteristics of cows with mild fatty liver have been reported previously (Du et al., 2018b).

Concentrations of glucose, BHB, and fatty acids in serum were determined using a Hitachi 7170 autoanalyzer (Hitachi, Tokyo, Japan) with commercially available kits (BHB: cat. no. RB1008; fatty acids: cat. no. FA115; glucose: cat. no. GL3815; Randox Laboratories, Crumlin, UK). The limits of quantification of glucose, BHB, and fatty acids were 0.200 to $35.5 \mathrm{mM}, 0.100$ to $5.75 \mathrm{mM}$, and 0.072 to $2.24 \mathrm{mM}$, respectively. Additionally, intra-assay $\mathrm{CV}$ for glucose, BHB and fatty acids were $<3.5 \%,<5 \%$, and $<5 \%$, respectively. Interassay CV for glucose, BHB, and fatty acids were $<10 \%$, $<10 \%$, and $<15 \%$, respectively.

For the determination of TG content, liver tissue $(\sim 20 \mathrm{mg})$ was homogenized in 5\% Triton-X100 (T8787; Sigma-Aldrich Co., St. Louis, MO) and then heated in a water bath $\left(85^{\circ} \mathrm{C}\right)$ for $3 \mathrm{~min}$. After the sample was cooled to room temperature, it was vortexed and centrifuged at $2,000 \times g$ for $5 \mathrm{~min}$ at $4^{\circ} \mathrm{C}$. The supernatant was collected and stored at $-80^{\circ} \mathrm{C}$ before analysis. The TG content was measured using an enzymatic kit (E1013; Applygen Technologies, Beijing, China) following the manufacturer's instructions. Total protein concentration was estimated with the bicinchoninic acid method (P1511; Applygen Technologies Inc.).

Western blotting was performed as described previously (Dessauge et al., 2011; Du et al., 2017b). Total protein was extracted from liver tissue using a commercial protein extraction kit (C510003; Sangon Biotech Co. Ltd., Shanghai, China), and the bicinchoninic acid assay performed to estimate the concentration of total protein. A total of $35 \mu \mathrm{g}$ of protein from each sample was separated by 12 or $15 \%$ SDS-PAGE. Then, gels were electrophoretically transferred to polyvinylidene difluoride membranes. Subsequently, 3\% BSA/ Tris-buffered saline/Tween (TBS-T) buffer was used to block the membranes at room temperature for 4 h. The blocked membranes were incubated overnight at $4^{\circ} \mathrm{C}$ with primary antibody against sequestosome- 1 
(SQSTM1, also called p62; 1:2,000, ab101266; Abcam, Cambridge, UK), microtubule-associated protein 1 light chain 3 (MAP1LC3, also called LC3; 1:1,000, ab48394; Abcam), $\beta$-actin (1:2,000, ab8226; Abcam), ubiquitin (1:200, sc-271289; Santa Cruz Biotechnology, Santa Cruz, CA), autophagy-related gene (ATG)5 (1:500; NB110-53818; Novus Biologicals, Littleton, CO), or ATG7 (1:1,000, D12B11; Cell Signaling Technology Inc., Danvers, MA). After incubation, the membranes were washed with TBS-T in an orbital shaker (18-1, Qilinbeier, Haimen, China), and then incubated with horseradish peroxidase-conjugated anti-rabbit or anti-mouse immunoglobulin at room temperature for $45 \mathrm{~min}$. Immunoreactive bands were visualized by enhanced chemiluminescence solution (Beyotime Biotechnology Inc., Shanghai, China). $\beta$-Actin was the internal control and the intensity of each target protein band was expressed relative to that of $\beta$-actin. Each sample was run in triplicate. Protein intensity was quantified using Image-Pro Plus 6.0 (Media Cybernetics, Bethesda, MD).

For the quantitative reverse-transcription (qRT) PCR assay, total RNA from liver tissue $(15 \mathrm{mg})$ was extracted using RNAiso Plus (9108, TaKaRa Biotechnology Co. Ltd., Dalian, China) according to the manufacturer's instructions. The RNA concentration and quality were measured using a K5500 microspectrophotometer (Beijing Kaiao Technology Development Ltd., Beijing, China) and electrophoresis (1\% agarose gels). According to MIQE guidelines, an optical density ratio at 260 and $280 \mathrm{~nm}\left(\mathrm{OD}_{260} / \mathrm{OD}_{280}\right)$ of 1.8 to 2.0 is an appropriate threshold of suitable RNA quality. In our study, the $\mathrm{OD}_{260} / \mathrm{OD}_{280}$ ratio of the total RNA averaged 1.9.

A total of $1 \mu \mathrm{g}$ of RNA from each sample was reverse-transcribed to $\mathrm{cDNA}$ in $25-\mu \mathrm{L}$ reactions using a reverse transcription kit (RR047A, TaKaRa Biotechnology Co. Ltd.) according to the supplier's protocol. We evaluated mRNA expression using qRT-PCR with the SYBR Green QuantiTect RT-PCR Kit (TaKaRa Biotechnology Co. Ltd.) and a 7500 Real-Time PCR System (Applied Biosystems/Thermo Fisher Scientific Inc., Waltham, MA).

The relative expression of each target gene was normalized to reference genes $\beta$-actin $(A C T B)$ and glyceraldehyde-3-phosphate dehydrogenase (GAPDH) and calculated using the $2^{-\Delta \Delta \mathrm{Ct}}$ method. The qRT$\mathrm{PCR}$ reaction was performed in triplicate for each cow and 15 cows were included per group. The validated primers used for SQSTM1, MAP1LC3, ATG5, and $A T G 7$ are shown in Supplemental Table S2 (https:/ /doi.org/10.3168/jds.2019-17457). The cycle threshold values of $A C T B$ and $G A P D H$ were not affected by liver fat content, which validates their usefulness as control genes (Connor et al., 2010; Morey et al., 2011; Du et al., 2017a; Supplemental Figure S1; https://doi.org/10 $.3168 /$ jds.2019-17457).

Each measurement in this study was repeated at least 3 times. Data were analyzed with SPSS 19.0 (IBM Corp., Armonk, NY) or GraphPad Prism 5.0 software (Graph Pad Software, San Diego, CA). In this study, data from Western blotting and qRT-PCR were normally distributed and analyzed using paired $t$-tests; other data were not normally distributed and were analyzed with Wilcoxon signed-rank test. Data are expressed as the mean \pm standard error of the mean (SEM); $P<$ 0.05 was considered statistically significant and $P<$ 0.01 was markedly significant compared with controls.

The baseline characteristics of control and mild fatty liver cows were reported by our previous study (Du et al., 2018b). Compared with controls, cows with mild fatty liver had numerically greater $(P>0.05) \mathrm{BW}$. Milk production and serum concentration of glucose were lower $(P<0.05)$ in cows with mild fatty liver. Body condition score was higher $(P<0.05)$ in cows with mild fatty liver. Serum concentrations of BHB and fatty acids were greater $(P<0.05$ and $P<0.01$, respectively) in cows with mild fatty liver, and the TG content $(\mathrm{g} / \mathrm{g}$ of wet weight) was greater $(P<0.01)$ in cows with mild fatty liver.

The abundance of ubiquitinated proteins was lower $(P<0.01)$ in the liver of cows with mild fatty liver (Figure 1A and B). The protein abundance of hepatic MAP1LC3 and SQSTM1 was lower $(P<0.01)$ in the liver of cows with mild fatty liver (Figure $2 \mathrm{~A}, \mathrm{~B}$ and $\mathrm{C}$ ). In contrast, the mRNA abundance of MAP1LC3 and SQSTM1 was greater $(P<0.01)$ in the liver of cows with mild fatty liver (Figure $2 \mathrm{D}$ and $\mathrm{E}$ ).

Hepatic protein abundance of ATG5 and ATG7 did not differ $(P>0.05)$ between mild fatty liver and healthy cows (Figure 3A, B, and C). However, mRNA abundance of $A T G 5$ and $A T G 7$ was greater $(P<0.01)$ in the liver of cows with mild fatty liver (Figure 3D and $\mathrm{E})$.

Even though previous studies investigated the adaptive mechanism of perinatal cows (Loor, 2010; Esposito et al., 2014; Ha et al., 2017), the underlying mechanism was still indistinct in dairy cows with mild fatty liver. Autophagy is an evolutionarily conserved adaptive mechanism that maintains cellular homeostasis and energy balance by degradation of cellular constituents or damaged organelles (Schneider and Cuervo, 2014). However, the interaction between autophagy and hepatic adaptations in postpartum dairy cows with mild fatty liver remains obscure. Here, we demonstrated that the formation and degradation of autophagosomes were enhanced in the liver of cows with mild fatty liver. These data suggested that autophagy might be 
involved in hepatic adaptive mechanisms during the onset of mild fatty liver.

Autophagy is a dynamic process, which begins with the formation of preautophagosomes that sequester targeted cell constituents and fuse with lysosomes to form autolysosomes in which the cargos are degraded (Nakatogawa et al., 2009; Parzych and Klionsky, 2014). During the process, ATG7 activates ATG5 (involved in preautophagosome formation) and forms a complex with ATG12 and ATG16 (Wei et al., 2019). Subsequently, LC3-I is cleaved by the protease ATG4 to form LC3-II, which remains on mature autophagosomes until after fusion with lysosomes and is commonly used to monitor autophagy. Ubiquitination of proteins or organelles is also required for their complete sequestration in autophagosomes (Allaire et al., 2019); SQSTM1 (p62) acts as an adaptor protein that interacts with LC3-II to target ubiquitinated cargos for autophagyspecific degradation (Grumati and Dikic, 2018).

Previous studies demonstrated that a greater abundance of ubiquitinated proteins and p62 reflects a blocked autophagic flux (González-Rodríguez et al., 2014; Du et al., 2018a). Conversely, high activity of autophagy promotes the degradation of ubiquitinated cargos and p62 (Pankiv et al., 2007). Thus, the lower abundance of ubiquitinated proteins and p62 in our study indicated that degradation of autophagosomes was enhanced during mild fatty liver. In addition, although the mRNA abundance of MAP1LC3 was higher, protein abundance of LC3-II was lower in mild fatty liver cows, further indicating enhanced degradation of autophagosomes. It is noteworthy that greater mRNA abundance of autophagosome formation molecules ATG5 and ATG7 was observed in mild fatty liver cows, suggesting enhanced formation of autophagosomes. However, the protein abundance of ATG5 and ATG7 was unchanged, which might result from enhanced degradation of autophagosomes. Altogether, the mRNA and protein changes of autophagic-regulated molecules in mild fatty liver cows indicated enhanced formation and degradation of autophagosomes, suggesting that autophagy may be involved in the onset of adaptive mechanisms of cows with mild fatty liver.

Under nutrient-deficient conditions, autophagic degradation of cellular constituents can efficiently recycle essential nutrients to sustain basic tissue functions (Filomeni et al., 2015). Accordingly, energy deficiency of mild fatty liver cows may explain the enhanced autophagosome formation and degradation. In addition, serum concentrations of BHB were greater in

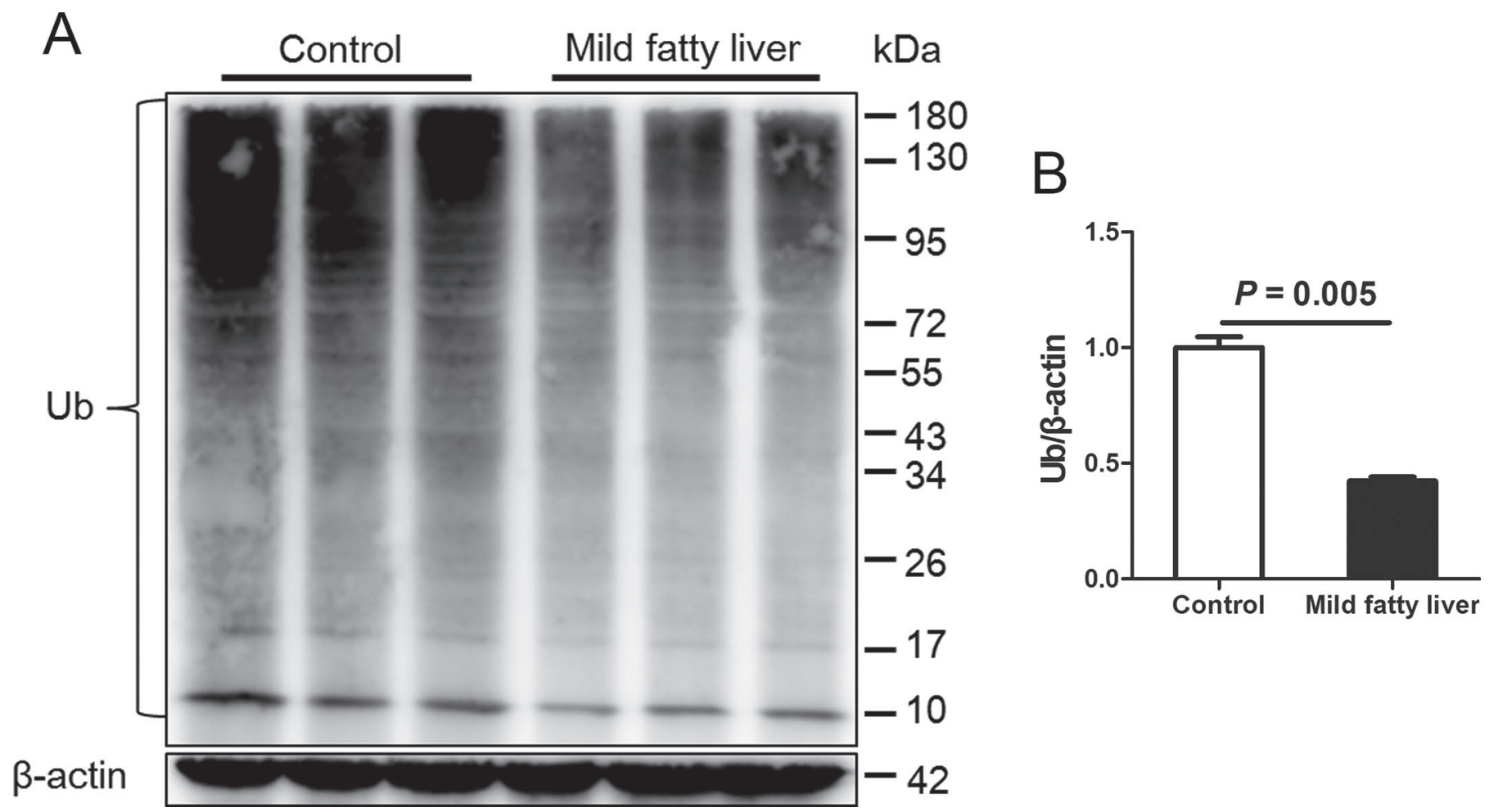

Figure 1. (A) Hepatic protein abundance of ubiquitinated (Ub) proteins in healthy cows $(\mathrm{n}=15)$ and dairy cows with mild fatty liver $(\mathrm{n}=$ 15). Representative blots are shown. (B) Quantification of hepatic abundance of ubiquitinated proteins. Data were analyzed with paired $t$-tests and expressed as mean \pm SEM. 
cows with mild fatty liver than in healthy cows. A previous study demonstrated that BHB could activate the AMP-activated protein kinase (AMPK) signaling pathway in bovine hepatocytes (Deng et al., 2015). Importantly, AMPK is an upstream regulator of autophagy and can markedly elevate the formation and degradation of autophagosomes (Gwinn et al., 2008). Therefore, the enhanced formation and degradation of autophagosomes in the liver of cows with mild fatty liver might result from increased BHB content. Peroxisome proliferator-activated receptor $\alpha(\mathbf{P P A R} \boldsymbol{\alpha})$ is a master transcription factor that regulates expression of $\beta$-oxidative genes (Qiu et al., 2008); nevertheless, growing evidence reveals a role for activated PPAR $\alpha$ in promoting autophagy as an adaptive mechanism under stress conditions (Kim et al., 2017). In line with the enhanced formation and degradation of autophagosomes in the liver of cows with mild fatty liver, our previous studies showed higher expression of PPAR $\alpha$ in mild fatty liver cows (Du et al., 2018b). Therefore, our results combined with those of previous studies revealed that upregulated PPAR $\alpha$ activities might be associated with enhanced autophagosome formation and degradation. In addition to the effects of energy status, BHB content, and PPAR $\alpha$ activity on autophagy, at least in nonruminants, other factors such as blood insulin level and intracellular calcium content have been shown to play a role in regulating autophagy (Harris, 2011; Medina and Ballabio, 2015). Accordingly, future studies in transition cows should examine the potential multifactorial control of this important cellular mechanism.

Impaired autophagy activity can lead to accumulation of toxic aggregates, such as misfolded or damaged proteins and mitochondria that can further increase the risk of cell death (Ravanan et al., 2017). Some evidence indicates that the normal increase in hepatic metabolic rate during the transition period (Reynolds et al., 2003) causes an increase in these cellular toxic aggregates (Bannink et al., 2012; Esposito et al., 2014). In addition to cell damage, experiments in human and mouse revealed that blocked autophagy induces oxidative stress and endoplasmic reticulum stress, and further exacer-
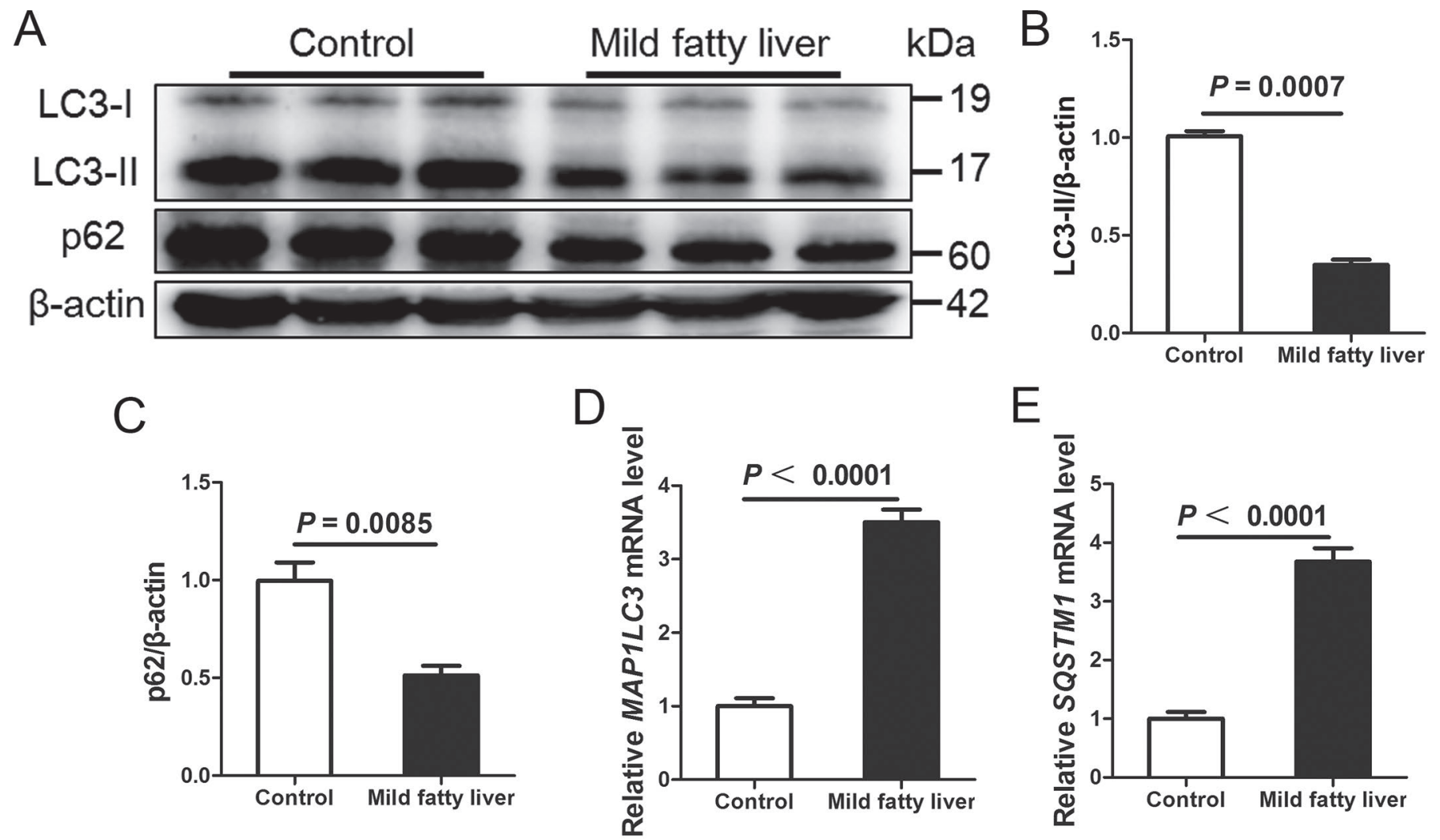

Figure 2. (A) Hepatic protein abundance of LC3 and p62 in healthy cows $(\mathrm{n}=15)$ and in dairy cows with mild fatty liver (n = 15). Representative blots are shown. (B, C) Quantification of hepatic protein abundance of LC3-II and p62. (D, E) Relative hepatic mRNA abundance of MAP1LC3 and SQSTM1 in healthy cows $(\mathrm{n}=15)$ and in dairy cows with mild fatty liver $(\mathrm{n}=15)$. Data were analyzed with paired $t$-tests and expressed as mean \pm SEM. Microtubule-associated protein 1 light chain 3 (LC3)-I, microtubule-associated protein 1 light chain 3 (LC3)-II, and sequestosome-1 (p62). 


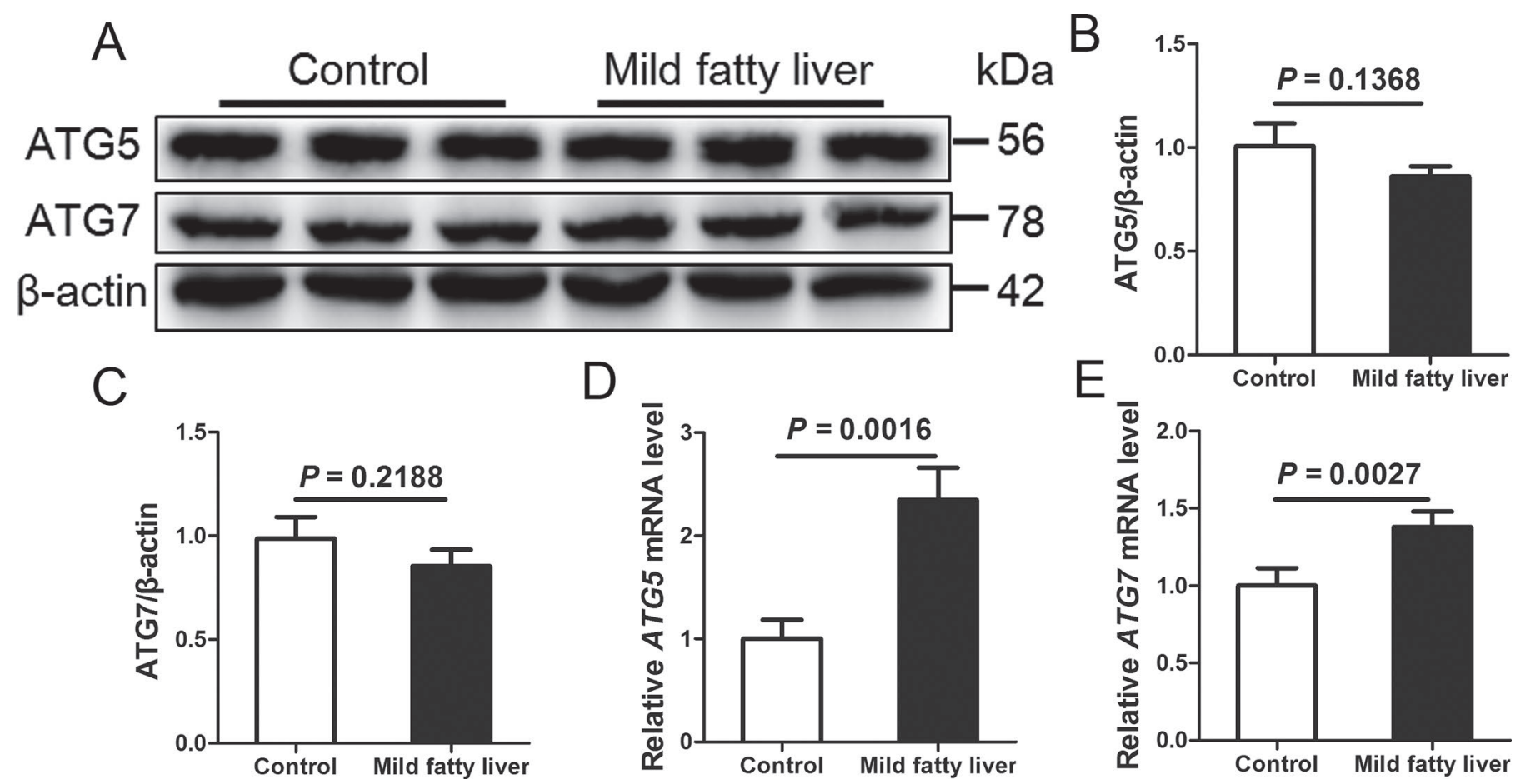

Figure 3. (A) Hepatic protein levels of ATG5 and ATG7 in healthy cows $(\mathrm{n}=15)$ and in dairy cows with mild fatty liver $(\mathrm{n}=15)$. Representative blots are shown. (B, C) Quantification of hepatic protein abundance of ATG5 and ATG7. (D, E) Relative hepatic mRNA abundance of $A T G 5$ and $A T G 7$ in healthy cows $(\mathrm{n}=15)$ and dairy cows with mild fatty liver $(\mathrm{n}=15)$. Data were analyzed with paired $t$-tests and expressed as mean \pm SEM. Autophagy-related gene (ATG) 5 and autophagy-related gene (ATG) 7 .

bates hepatic steatosis and injury (Ding et al., 2010; Ma et al., 2013; Tanaka et al., 2016). Therefore, the enhanced formation and degradation of autophagosomes in cows with mild fatty liver indicated that autophagy makes an important contribution to maintaining energy homeostasis and preventing progression of fatty liver in dairy cows.

In conclusion, the hepatic mRNA abundance of the autophagosome formation-related molecules $M A$ P1LC3, ATG5, and ATG7 was greater, whereas the abundance of ubiquitinated proteins and of LC3-II and p62 protein were lower in the liver of cows with mild fatty liver, suggesting that the formation and degradation of autophagosomes is enhanced in the liver of cows with mild fatty liver. These findings shed light on an underappreciated function of autophagy in adaptive processes within the liver of dairy cows during the transition period; this adaptive process ensures the physical condition of perinatal dairy cows.

\section{ACKNOWLEDGMENTS}

This work was supported by the National Key Research and Development Program (Beijing, China; grant no. 2016YFD0501206), the National Natural Science Foundation of China (Beijing, China; grant no.
31772810), Natural Science Foundation of Jilin Province (Changchun, China; grant no. 20170101148JC), Natural Science Scientific Research Project Jointly Established by Jilin University and Jilin Province (Changchun, China; grant no. SXGJSF2017-6), the Talents Cultivation Program of Jilin University, and China Postdoctoral Science Foundation (2019TQ0115). The authors have not stated any conflicts of interest.

\section{REFERENCES}

Allaire, M., P. E. Rautou, P. Codogno, and S. Lotersztajn. 2019. Autophagy in liver diseases: Time for translation? J. Hepatol. 70:985998. https://doi.org/10.1016/j.jhep.2019.01.026.

Bannink, A., W. J. J. Gerrits, J. France, and J. Dijkstra. 2012. Variation in rumen fermentation and the rumen wall during the transition period in dairy cows. Anim. Feed Sci. Technol. 172:80-94. https://doi.org/10.1016/j.anifeedsci.2011.12.010.

Bobe, G., J. W. Young, and D. C. Beitz. 2004. Invited review: Pathology, etiology, prevention, and treatment of fatty liver in dairy cows. J. Dairy Sci. 87:3105-3124. https://doi.org/10.3168/jds .S0022-0302(04)73446-3.

Ceciliani, F., C. Lecchi, C. Urh, and H. Sauerwein. 2018. Proteomics and metabolomics characterizing the pathophysiology of adaptive reactions to the metabolic challenges during the transition from late pregnancy to early lactation in dairy cows. J. Proteomics 178:92-106. https://doi.org/10.1016/j.jprot.2017.10.010.

Connor, E. E., R. L. Baldwin, A. V. Capuco, C. M. Evock-Clover, S. E. Ellis, and K. S. Sciabica. 2010. Characterization of glucagonlike peptide 2 pathway member expression in bovine gastrointes- 
tinal tract. J. Dairy Sci. 93:5167-5178. https://doi.org/10.3168/ jds.2010-3205.

Deng, Q., G. Liu, L. Liu, Y. Zhang, L. Yin, X. Shi, J. Wang, X. Yuan, G. Sun, Y. Li, W. Yang, L. Guo, R. Zhang, Z. Wang, X. Li, and X. Li. 2015. BHBA influences bovine hepatic lipid metabolism via AMPK signaling pathway. J. Cell. Biochem. 116:1070-1079. https: //doi.org/10.1002/jcb.25062.

Dessauge, F., V. Lollivier, B. Ponchon, R. Bruckmaier, L. Finot, S. Wiart, E. Cutullic, C. Disenhaus, S. Barbey, and M. Boutinaud. 2011. Effects of nutrient restriction on mammary cell turnover and mammary gland remodeling in lactating dairy cows. J. Dairy Sci. 94:4623-4635. https://doi.org/10.3168/jds.2010-4012.

Ding, W. X., M. Li, X. Chen, H. M. Ni, C. W. Lin, W. Gao, B. Lu, D. B. Stolz, D. L. Clemens, and X. M. Yin. 2010. Autophagy reduces acute ethanol-induced hepatotoxicity and steatosis in mice. Gastroenterology 139:1740-1752. https://doi.org/10.1053/j.gastro 2010.07.041.

Du, X., L. Chen, D. Huang, Z. Peng, C. Zhao, Y. Zhang, Y. Zhu, Z. Wang, X. Li, and G. Liu. 2017a. Elevated apoptosis in the liver of dairy cows with ketosis. Cell. Physiol. Biochem. 43:568-578. https: //doi.org/10.1159/000480529.

Du, X., G. Liu, J. J. Loor, Z. Fang, R. Bucktrout, Y. Yang, Q. Ye, Z. Shi, T. Shen, X. Wang, Z. Peng, C. Zhao, B. Lv, D. Xing, Y. Zhu, X. Li, and X. Li. 2018a. Impaired hepatic autophagic activity in dairy cows with severe fatty liver is associated with inflammation and reduced liver function. J. Dairy Sci. 101:11175-11185. https:/ /doi.org/10.3168/jds.2018-15120.

Du, X., T. Shen, H. Wang, X. Qin, D. Xing, Q. Ye, Z. Shi, Z. Fang, Y. Zhu, Y. Yang, Z. Peng, C. Zhao, B. Lv, X. Li, G. Liu, and X. Li. 2018b. Adaptations of hepatic lipid metabolism and mitochondria in dairy cows with mild fatty liver. J. Dairy Sci. 101:9544-9558. https://doi.org/10.3168/jds.2018-14546.

Du, X., Z. Shi, Z. Peng, C. Zhao, Y. Zhang, Z. Wang, X. Li, G. Liu, and X. Li. 2017b. Acetoacetate induces hepatocytes apoptosis by the ROS-mediated MAPKs pathway in ketotic cows. J. Cell. Physiol. 232:3296-3308. https://doi.org/10.1002/jcp.25773.

Esposito, G., P. C. Irons, E. C. Webb, and A. Chapwanya. 2014. Interactions between negative energy balance, metabolic diseases, uterine health and immune response in transition dairy cows. Anim. Reprod. Sci. 144:60-71. https://doi.org/10.1016/j.anireprosci.2013 .11 .007 .

FASS. 2010. Guidelines for the Care and Use of Agricultural Animals in Research and Teaching. 3rd ed. FASS Inc., Champaign, IL.

Filomeni, G., D. De Zio, and F. Cecconi. 2015. Oxidative stress and autophagy: The clash between damage and metabolic needs. Cell Death Differ. 22:377-388. https://doi.org/10.1038/cdd.2014.150.

González-Rodríguez, A., R. Mayoral, N. Agra, M. P. Valdecantos, V. Pardo, M. E. Miquilena-Colina, J. Vargas-Castrillon, O. Lo Iacono, M. Corazzari, G. M. Fimia, M. Piacentini, J. Muntane, L. Bosca, C. Garcia-Monzon, P. Martin-Sanz, and A. M. Valverde. 2014. Impaired autophagic flux is associated with increased endoplasmic reticulum stress during the development of NAFLD. Cell Death Dis. 5:e1179. https://doi.org/10.1038/cddis.2014.162.

Grumati, P., and I. Dikic. 2018. Ubiquitin signaling and autophagy. J. Biol. Chem. 293:5404-5413. https://doi.org/10.1074/jbc.TM117 .000117 .

Gwinn, D. M., D. B. Shackelford, D. F. Egan, M. M. Mihaylova, A. Mery, D. S. Vasquez, B. E. Turk, and R. J. Shaw. 2008. AMPK phosphorylation of raptor mediates a metabolic checkpoint. Mol. Cell 30:214-226. https://doi.org/10.1016/j.molcel.2008.03.003.

Ha, N. T., C. Drogemuller, C. Reimer, F. Schmitz-Hsu, R. M. Bruckmaier, H. Simianer, and J. J. Gross. 2017. Liver transcriptome analysis reveals important factors involved in the metabolic adaptation of the transition cow. J. Dairy Sci. 100:9311-9323. https:// doi.org/10.3168/jds.2016-12454.

Harris, J. 2011. Autophagy and cytokines. Cytokine 56:140-144. https: //doi.org/10.1016/j.cyto.2011.08.022.

Imhasly, S., C. Bieli, H. Naegeli, L. Nystrom, M. Ruetten, and C. Gerspach. 2015. Blood plasma lipidome profile of dairy cows during the transition period. BMC Vet. Res. 11:252. https://doi.org/10 $.1186 / \mathrm{s} 12917-015-0565-8$.
Ingvartsen, K. L. 2006. Feeding- and management-related diseases in the transition cow: Physiological adaptations around calving and strategies to reduce feeding-related diseases. Anim. Feed Sci. Technol. 126:175-213. https://doi.org/10.1016/j.anifeedsci.2005 .08 .003 .

Juárez-Hernández, E., N. C. Chavez-Tapia, M. Uribe, and V. J. Barbero-Becerra. 2016. Role of bioactive fatty acids in nonalcoholic fatty liver disease. Nutr. J. 15:72. https://doi.org/10.1186/s12937 $-016-0191-8$.

Kim, K. H., and M. S. Lee. 2014. Autophagy - A key player in cellular and body metabolism. Nat. Rev. Endocrinol. 10:322-337. https:// doi.org/10.1038/nrendo.2014.35.

Kim, Y. S., H. M. Lee, J. K. Kim, C. S. Yang, T. S. Kim, M. Jung, H. S. Jin, S. Kim, J. Jang, G. T. Oh, J. M. Kim, and E. K. Jo. 2017. PPAR-alpha activation mediates innate host defense through induction of TFEB and lipid catabolism. J. Immunol. 198:32833295. https://doi.org/10.4049/jimmunol.1601920.

Lavallard, V. J., and P. Gual. 2014. Autophagy and non-alcoholic fatty liver disease. BioMed Res. Int. 2014:120179. https://doi.org/ 10.1155/2014/120179.

Litherland, N. B., H. M. Dann, and J. K. Drackley. 2011. Prepartum nutrient intake alters palmitate metabolism by liver slices from peripartal dairy cows. J. Dairy Sci. 94:1928-1940. https://doi.org/ 10.3168/jds.2010-3220.

Loor, J. J. 2010. Genomics of metabolic adaptations in the peripartal cow. Animal 4:1110-1139. https://doi.org/10.1017/ S1751731110000960.

Loor, J. J., M. Bionaz, and J. K. Drackley. 2013. Systems physiology in dairy cattle: Nutritional genomics and beyond. Annu. Rev. Anim. Biosci. 1:365-392. https://doi.org/10.1146/annurev-animal $-031412-103728$.

Ma, D., M. M. Molusky, J. Song, C. R. Hu, F. Fang, C. Rui, A. V. Mathew, S. Pennathur, F. Liu, J. X. Cheng, J. L. Guan, and J. D. Lin. 2013. Autophagy deficiency by hepatic FIP200 deletion uncouples steatosis from liver injury in NAFLD. Mol. Endocrinol. 27:1643-1654. https://doi.org/10.1210/me.2013-1153.

Medina, D. L., and A. Ballabio. 2015. Lysosomal calcium regulates autophagy. Autophagy 11:970-971. https://doi.org/10.1080/ 15548627.2015.1047130.

Morey, S. D., L. K. Mamedova, D. E. Anderson, C. K. Armendariz, E. C. Titgemeyer, and B. J. Bradford. 2011. Effects of encapsulated niacin on metabolism and production of periparturient dairy cows. J. Dairy Sci. 94:5090-5104. https://doi.org/10.3168/jds.2011 -4304 .

Nakatogawa, H., K. Suzuki, Y. Kamada, and Y. Ohsumi. 2009. Dynamics and diversity in autophagy mechanisms: Lessons from yeast. Nat. Rev. Mol. Cell Biol. 10:458-467. https://doi.org/10 $.1038 / \mathrm{nrm} 2708$.

Pankiv, S., T. H. Clausen, T. Lamark, A. Brech, J. A. Bruun, H. Outzen, A. Overvatn, G. Bjorkoy, and T. Johansen. 2007. p62/ SQSTM1 binds directly to Atg8/LC3 to facilitate degradation of ubiquitinated protein aggregates by autophagy. J. Biol. Chem. 282:24131-24145. https://doi.org/10.1074/jbc.M702824200.

Parzych, K. R., and D. J. Klionsky. 2014. An overview of autophagy: Morphology, mechanism, and regulation. Antioxid. Redox Signal. 20:460-473. https://doi.org/10.1089/ars.2013.5371.

Reynolds, C. K., P. C. Aikman, B. Lupoli, D. J. Humphries, and D. E. Beever. 2003. Splanchnic metabolism of dairy cows during the transition from late gestation through early lactation. J. Dairy Sci. 86:1201-1217. https://doi.org/10.3168/jds.S0022-0302(03)73704 $-7$.

Qin, N., T. Kokkonen, S. Salin, T. Seppanen-Laakso, J. Taponen, A. Vanhatalo, and K. Elo. 2018. Prepartal high-energy feeding with grass silage-based diets does not disturb the hepatic adaptation of dairy cows during the periparturient period. J. Dairy Sci. 101:8929-8943. https://doi.org/10.3168/jds.2017-13153.

Qiu, L., X. Wu, J. F. Chau, I. Y. Szeto, W. Y. Tam, Z. Guo, S. K. Chung, P. J. Oates, S. S. Chung, and J. Y. Yang. 2008. Aldose reductase regulates hepatic peroxisome proliferator-activated receptor alpha phosphorylation and activity to impact lipid homeo- 
stasis. J. Biol. Chem. 283:17175-17183. https://doi.org/10.1074/ jbc.M801791200.

Ravanan, P., I. F. Srikumar, and P. Talwar. 2017. Autophagy: The spotlight for cellular stress responses. Life Sci. 188:53-67. https:// doi.org/10.1016/j.lfs.2017.08.029.

Schneider, J. L., and A. M. Cuervo. 2014. Liver autophagy: Much more than just taking out the trash. Nat. Rev. Gastroenterol. Hepatol. 11:187-200. https://doi.org/10.1038/nrgastro.2013.211.

Tanaka, S., H. Hikita, T. Tatsumi, R. Sakamori, Y. Nozaki, S. Sakane, Y. Shiode, T. Nakabori, Y. Saito, N. Hiramatsu, K. Tabata, T. Kawabata, M. Hamasaki, H. Eguchi, H. Nagano, T. Yoshimori, and T. Takehara. 2016. Rubicon inhibits autophagy and accelerates hepatocyte apoptosis and lipid accumulation in nonalcoholic fatty liver disease in mice. Hepatology 64:1994-2014. https://doi .org/10.1002/hep. 28820
Wei, P. L., C. Y. Huang, and Y. J. Chang. 2019. Propyl gallate inhibits hepatocellular carcinoma cell growth through the induction of ROS and the activation of autophagy. PLoS One 14:e0210513. https://doi.org/10.1371/journal.pone.0210513.

\section{ORCIDS}

Juan J. Loor @ https://orcid.org/0000-0003-1586-4365

Yusheng Liang ๑ https://orcid.org/0000-0003-1751-6823

Taiyu Shen $\odot$ https://orcid.org/0000-0002-0257-8741

Guowen Liu (i) https://orcid.org/0000-0002-2672-6104

Xiaobing Li 우 https://orcid.org/0000-0001-9105-6365

Xinwei Li ๑ https://orcid.org/0000-0002-0765-677X 